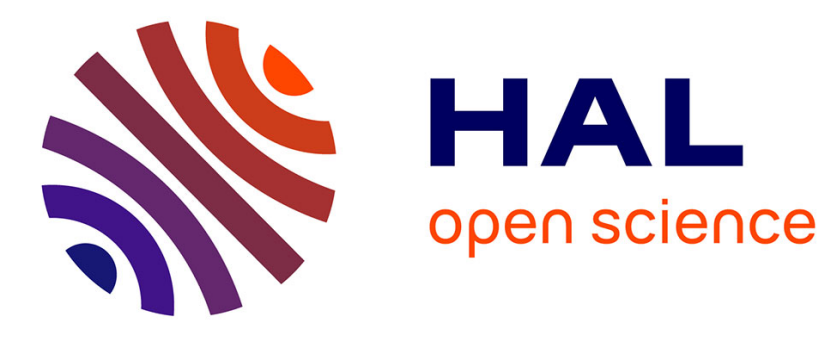

\title{
Theory of Talbot lasers
}

\author{
H. Guillet de Chatellus, E. Lacot, W. Glastre, O. Jacquin, O. Hugon
}

\section{To cite this version:}

H. Guillet de Chatellus, E. Lacot, W. Glastre, O. Jacquin, O. Hugon. Theory of Talbot lasers. Physical Review A : Atomic, molecular, and optical physics [1990-2015], 2013, 88, pp.33828. 10.1103/PHYSREVA.88.033828 . hal-00951890

\section{HAL Id: hal-00951890 https://hal.science/hal-00951890}

Submitted on 27 Feb 2014

HAL is a multi-disciplinary open access archive for the deposit and dissemination of scientific research documents, whether they are published or not. The documents may come from teaching and research institutions in France or abroad, or from public or private research centers.
L'archive ouverte pluridisciplinaire HAL, est destinée au dépôt et à la diffusion de documents scientifiques de niveau recherche, publiés ou non, émanant des établissements d'enseignement et de recherche français ou étrangers, des laboratoires publics ou privés. 


\title{
Theory of Talbot lasers
}

\author{
H. Guillet de Chatellus, E. Lacot, W. Glastre, O. Jacquin and O. Hugon* \\ CNRS/Univ. Grenoble 1, Laboratoire Interdisciplinaire de Physique, UMR 5588, Grenoble, F-38041, France
}

(Dated: June 4, 2013)

\begin{abstract}
We provide a theoretical study of frequency shifted feedback (FSF) lasers - i.e. lasers with an internal frequency shifter- seeded with a monochromatic wave. The resulting spectrum consists in a set of equidistant modes -labeled by $n$ - whose phases vary quadratically with $n$. We prove the emergence of a temporal fractional Talbot effect, leading to generation of Fourier transform-limited pulses at a repetition rate tunable by the parameters of the FSF cavity (cavity length and frequency shift per roundtrip), and limited by the spectral bandwidth of the laser. We characterize in detail the output field of this so-called "Talbot laser", and emphasize its specific intensity fluctuations. We evidence connections with some aspects of number theory by the appearance of Gauss sums and theta series in the expression of the laser field. Our predictions are in full agreement with the experimental results published in Guillet de Chatellus et al., Opt. Exp. (2013), in press. Practical applications and limitations are discussed.
\end{abstract}

PACS numbers: 42.25.Fx, 42.30.Kq, 42.60.Fc,

Diffraction is a fundamental concept in optics. In the far field of a grating shined with a monochromatic wave, the waves diffracted by the slits can be considered as plane waves with constant relative phase-shifts. When the latter is a multiple of $2 \pi$, constructive interferences occur and the light intensity is maximum. In the near field however, things are more subtle and inferring the diffracted pattern requires to consider the spherical nature of the waves diffracted by the slits. Talbot reported in 1836 a rich variety of phenomena in the vicinity of the grating: the repetition of the image of the diffraction grating at distances multiples of the so-called Talbot length, and most important for our point, the appearance at fractional distances of the Talbot length, of light fringes with a spatial frequency equal to (possibly large) multiples of the fundamental frequency of the grating $[1,2]$. The Talbot effect was later simply explained by the interference of waves in the paraxial approximation, i.e. showing quadratic phases [3, 4]. Similar behaviors are also encountered in wave packets revivals in quantum systems [5-8]: when the energy levels $E_{n}$ of the eigenmodes of a quantum system have a small quadratic (or higher order) dependence with $n$, the dynamics of the system exhibits both periodic revivals of the whole wavepacket and fractional revivals, that is the appearance of multiple mini-wavepackets or clones. This phenomenon, like the Talbot effect, is a particular illustration of sums with quadratic phase-shifts and connect to the mathematical properties of Gauss sums or Jacobi theta sums [9-11]. Note that the interest for physical systems showing quadratic phase shifts have recently been boosted by the demonstration of physical protocols for factoring large numbers [12-17].

It is interesting to reconsider the equivalent of the frac-

*Electronic address: hugues.guilletdechatellus@ujf-grenoble. fr tional Talbot effect in the time domain. A frequency comb of modelocked optical modes is the temporal equivalent of a grating of slits in the far field: constructive interferences occur periodically with time, generating pulses at a repetition rate equal to the mode spacing of the comb. Then in the time domain the fractional Talbot effect corresponds to pulsing at (possibly high) multiples of the mode spacing (fig. 1) and constitutes therefore an exciting and promising challenge: applications of ultrahigh repetition rate lasers concern the generation of $\mathrm{THz}$ waves, transfer of clock signals between remote users, optical sampling, optical cadencers for analog-todigital converters and ultrafast optical digital communications [18] as well as spectroscopy of metallic nanoparticles $[19,20]$. By analogy to the space domain, the demonstration of a laser showing fractional Talbot effect requires a comb of modes -that is monochromatic waves with a constant frequency spacing- with quadratic phases. A first solution has been provided by engineering the dispersion undergone by a modelocked laser in a specially designed Bragg gratings, so as to induce quadratic phases to the modes of the comb [21, 22]. This technique has been successfully applied and lead to multiplication of the repetition rate by a factor up to 10 [23]. However it is technically challenging to increase further the repetition rate and moreover the dispersion is fixed and the repetition rate is not easily tunable.

Interestingly a solution is brought naturally by a frequency shifted feedback (FSF) laser seeded with a monochromatic wave [24, 25]. A FSF laser is a cavity closed on the first diffraction order of an acousto-optics frequency-shifter (AOFS): each time a photon makes a roundtrip in the cavity, it undergoes a constant frequency shift. Typical diffraction efficiencies of AOFSs reach 90 $\%$. The intracavity field consists therefore in a frequency comb, with a mode spacing equal to the frequency shift per roundtrip. However contrary to mode-locked frequency combs where all modes share the same (or a linear) phase, the phases of the modes of the FSF laser 
a)

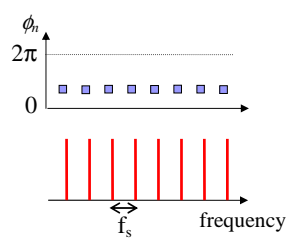

b)

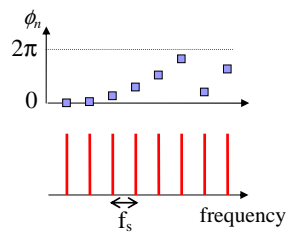

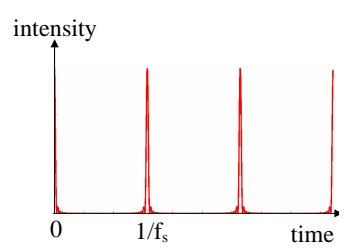

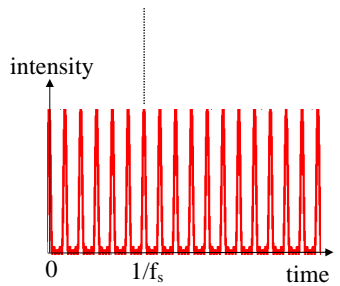

FIG. 1: Temporal fractional Talbot effect by the interference of monochromatic waves with a constant frequency spacing $f_{s}$. a): when the waves have constant (or linear) phases, pulses are generated at repetition rate $f_{s}$. b): when the waves have quadratic phases the repetition rate is multiplied by an integer, depending on the curvature of the parabola.

are quadratic. However this property has been somehow unexploited: most research on seeded FSF laser has focussed so far on the spectral characteristics of this source to realize optical synthesizers and frequency combs [2629].

More precisely regarding the temporal properties of the light emitted by seeded FSF lasers, early work on both passive and active FSF cavities reported the possibility to generate modelocked frequency combs when the frequency shift is a multiple of the cavity free spectral range $[24,30]$. A more recent theoretical work has shown numerically the possibility of increasing the repetition rate by adjusting the frequency shift and the cavity free spectral range as the ratio of two integers [31]. Very recently, our team provided an experimental demonstration of this concept by injecting a dye FSF cavity with a dye single mode seed laser: the generation of 6 ps Fourier transform-limited pulses was demonstrated with repetition rates tunable by steps of $80 \mathrm{MHz}$ between 0.24 and $36.6 \mathrm{GHz}$, that is over two orders of magnitude [32]. Here we explain this result in depth by providing an extensive description of the fractional Talbot effect in CW-seeded FSF lasers [3]. In the first part of the manuscript we introduce a simple model of a CW-seeded FSF laser. Then we consider the case where the optical spectrum of the CW-seeded FSF laser consists in a set of $\mathrm{N}$ optical modes with the same amplitude showing a constant frequency spacing and quadratic phases. We derive an expression of the resulting electric field and intensity and show that, depending on the curvature of the parabolic phases, it is possible to generate a temporal fractional Talbot effect, i.e. the generation of Fourier transform limited pulses with a tunable repetition rate. We characterize the pulses and the intensity fluctuations with respect to
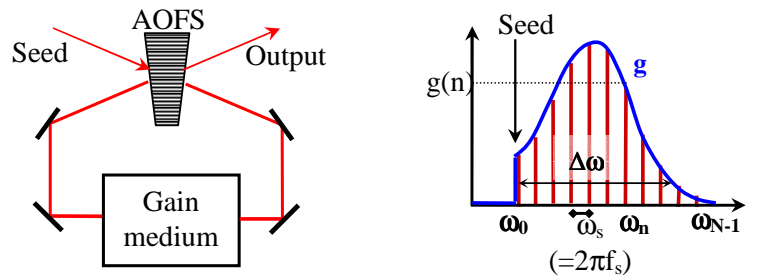

FIG. 2: Left: generic sketch of a CW-seeded FSF laser. The seed is injected in the cavity through the zero diffraction (i.e. undiffracted) order of the AOFS. The output field is extracted the same way. Right: resulting spectrum.

the curvature of the parabolic phases and show the emergence of self-similarity when the number of modes is increased. Our calculations are closely related to properties of Gauss sums in number theory and theoretical aspects of spatial Talbot effect. In the third part we consider a more realistic description of the CW-seeded FSF laser by taking into account the spectral shape arising from the gain et losses of the cavity and we derive generic expressions for the output intensity and its fluctuations. Finally we discuss possible extensions of this work for generating ultra-stable ultrahigh repetition rates in broadband CW-seeded FSF lasers and underline the fundamental limitations and the related technical requirements.

\section{THE CW INJECTION-SEEDED FSF LASER}

We consider a FSF cavity characterized by the roundtrip time $\tau_{c}=1 / f_{c}=2 \pi / \omega_{c}$ and the frequency shift per roundtrip $f_{s}=\omega_{s} / 2 \pi$ (fig. 2 ).

The cavity is injected continuously by the seeding field $E_{0} e^{-i \omega_{0}\left(t+\tau_{c}\right)}$. (The choice of the phase is made arbitrarily to simplify the following expressions.) At each roundtrip in the cavity the angular frequency is shifted by $\omega_{s}$. A leak of the AOFS on the zero order enables to extract a fraction of the intracavity field. The output spectrum consists in a comb of optical modes separated by the frequency $f_{s}$. The angular frequency of the mode $n$ (i.e. after $n$ roundtrips) is $\omega_{0}+n \omega_{s}$.

We define $\eta$ as the diffraction efficiency in amplitude of the AOFS and neglect the dependence of $\eta$ with the frequency. We consider the dependence of the gain medium in the cavity with the frequency by defining $h(n)$ as the single-pass gain in the laser medium of a optical mode at angular frequency $\omega_{0}+n \omega_{s}$. The resulting field at the output of the AOFS is therefore: 


$$
\begin{aligned}
E(t) & =E_{0}(1-\eta)^{2} h(0) e^{-i \omega_{0} t} \\
& +E_{0} \eta(1-\eta)^{2} h(0) h(1) e^{-i\left(\omega_{0}+\omega_{s}\right) t} e^{i \phi_{1}} \\
& +E_{0} \eta^{2}(1-\eta)^{2} h(0) h(1) h(2) e^{-i\left(\omega_{0}+2 \omega_{s}\right) t} e^{i \phi_{2}} \\
& +E_{0} \eta^{3}(1-\eta)^{2} h(0) h(1) h(2) h(3) e^{-i\left(\omega_{0}+3 \omega_{s}\right) t} e^{i \phi_{3}} \\
& +\ldots
\end{aligned}
$$

with $\phi_{1}=\left(\omega_{0}+\omega_{s}\right) \tau_{c}, \phi_{2}=\phi_{1}+\left(\omega_{0}+2 \omega_{s}\right) \tau_{c}, \phi_{3}=\phi_{2}+$ $\left(\omega_{0}+3 \omega_{s}\right) \tau_{c} \ldots$ Therefore $\phi_{n}=n \omega_{0} \tau_{c}+n(n+1) \omega_{s} \tau_{c} / 2$.

We define $g(n)=\eta^{n}(1-\eta)^{2} \Pi_{k=0}^{n} h(k) . g$ can therefore be seen as the envelope of the optical spectrum of the laser resulting from the successive gain and losses of the FSF cavity (fig. 2). We set $g(n)=0$ when $n<0$ and $n>N$, the cutoff limit of $g$.

The expression of the electric field at the output of the FSF laser seeded by a monochromatic wave is:

$$
E(t)=E_{0} e^{-i \omega_{0} t} \sum_{n} g(n) e^{-i n \omega_{s} t} e^{i n \omega_{0} \tau_{c}} e^{i \frac{n(n+1)}{2} \phi}
$$

where $\phi=\omega_{s} \tau_{c}=2 \pi f_{s} / f_{c}$. Note the quadratic dependence of the phases of the modes $[24,31]$.

\section{INTERFERENCE OF N OPTICAL MODES OF CONSTANT AMPLITUDE WITH QUADRATIC PHASES}

In this section, we treat the ideal case where the laser output consists in a set on $N$ modes with identical amplitude. This calculation is closely related to [3] and leads to interesting connections to number theory.

Setting $g$ as a top-hat function of width $N$ and amplitude unity, the electric field has the following expression:

$$
E(t)=E_{0} e^{-i \omega_{0} t} \sum_{n=0}^{N-1} e^{-i n \omega_{s} t} e^{i n \omega_{0} \tau_{c}} e^{i \frac{n(n+1)}{2} \phi}
$$

where $\phi=\omega_{s} \tau_{c}=2 \pi f_{s} / f_{c}$. Here $E(t)$ appears as a Fourier series with terms of equal amplitude and quadratic phases. Since $\omega_{0}$ is orders of magnitude larger than $\omega_{s}$, we consider the frame rotating at $\omega_{0}$ where we study the envelope of electric field. Writing $\theta=$ $\omega_{s} t-\omega_{0} \tau_{c}$, the electric field of the FSF laser is described by the $2 \pi$-periodic function:

$$
F(\theta)=\sum_{n=0}^{N-1} e^{-i n \theta} e^{i \frac{n(n+1)}{2} \phi} .
$$

It is noteworthy that the electric field can be plotted in the complex plane as a chain of $N$ phasors with quadratic phases. The resulting patterns consist in the repetition of Cornu spirals, called "curlicues" and evolving with time (fig. 3) [33].

In the following we study the properties of this function of the normalized time $\theta$ depending on the values of $\phi$.
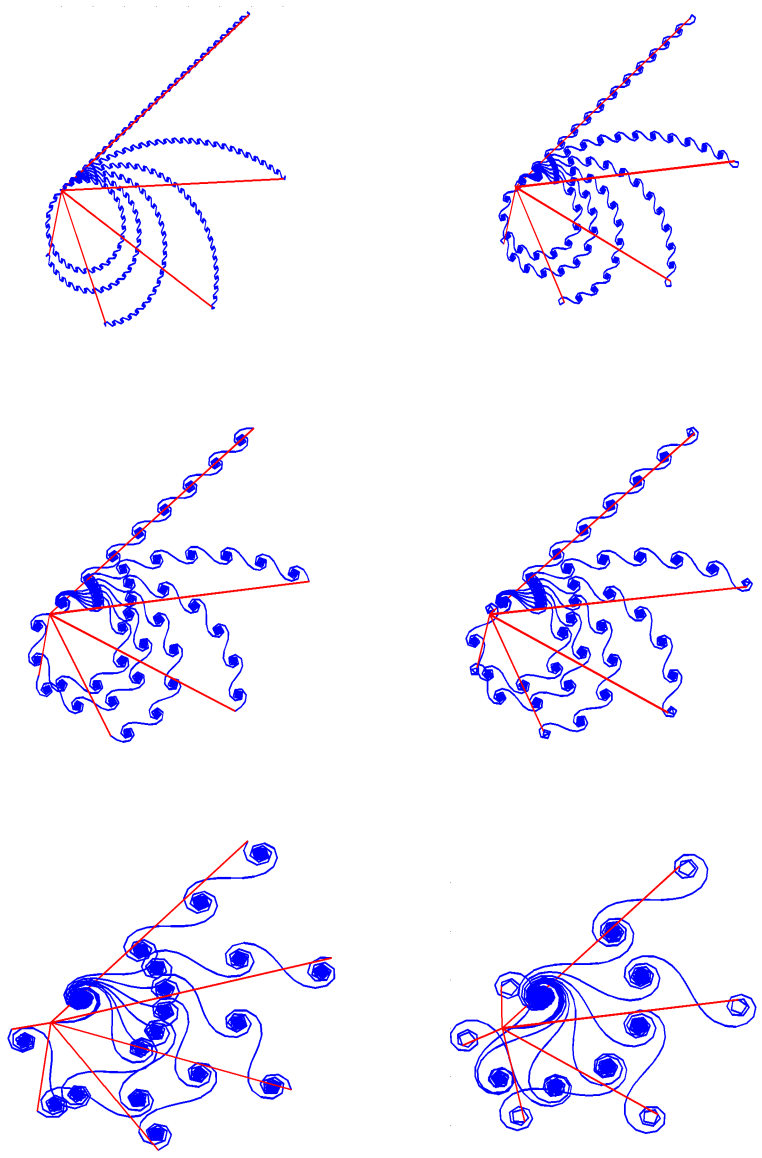

FIG. 3: Representation in the complex plane of $F(\theta)$ as a sum of $N=200$ phasors with quadratic phases. We set $\phi=2 \pi p / q$. In all six cases, $p=1$ and $q=7,15,25,27,51,89$ from left to right and top to bottom. For each value of $\phi$, we plot $F\left(\theta_{k}\right)$ for six consecutive values of $\theta: \theta_{k}=2 k \pi / 1000$ with $0 \leq k \leq 5$. The resulting total field corresponds to the straight line and evolves clockwise with $k$.

\section{A. Expression of the electric field}

We consider the specific case where $\phi=2 \pi p / q$ ( $p$ and $q$ coprimes) and $q<N$. Then the phases of the modes are given by $\phi_{n}=\pi n(n+1) p / q$ and we study $F(\theta)$ depending on the parity of integers $p$ and $q$. We define $K$ as the largest integer satisfying $N=K q+r, r$ integer.

\section{Case $p$ even or $q$ odd}

When $p$ is even or when $q$ is odd, one has:

$$
\begin{aligned}
\phi_{n+q} & =\pi\left(n^{2}+2 n q+q^{2}+n+q\right) p / q \\
& =\phi_{n}+2 \pi n p+\pi p(q+1)=\phi_{n} .
\end{aligned}
$$

$\phi_{n}$ is periodic with a period equal to $q$. Therefore: 


$$
\begin{aligned}
F(\theta) & =\sum_{n=0}^{K q} e^{-i n \theta} e^{i \phi_{n}}+\sum_{n=K q+1}^{N-1} e^{-i n \theta} e^{i \phi_{n}} \\
& =\sum_{n=0}^{q-1} e^{-i n \theta} e^{i \phi_{n}} \sum_{k=0}^{K-1} e^{-i k q \theta}+\sum_{n=K q+1}^{N-1} e^{-i n \theta} e^{i \phi_{n}} .
\end{aligned}
$$

The geometric series can be easily calculated and finally:

$$
F(\theta)=\frac{1-e^{-i K q \theta}}{1-e^{-i q \theta}} \sum_{n=0}^{q-1} e^{-i n \theta} e^{i \phi_{n}}+\sum_{n=K q+1}^{N-1} e^{-i n \theta} e^{i \phi_{n}}
$$

In the following we suppose that $q$ divides $N(N=K q)$ and therefore the second term on the right hand of the equation vanishes. Note that in the general case where $q$ does not divide $N$ and for a large value of $K(r \ll K q)$, the contribution of this term becomes indeed negligible (this point is discussed at the end of this section). Therefore the field reduces to:

$$
F(\theta)=\frac{1-e^{-i N \theta}}{1-e^{-i q \theta}} \sum_{n=0}^{q-1} e^{-i n \theta} e^{i \phi_{n}} .
$$

The intensity of the laser is:

$$
I(\theta)=|F(\theta)|^{2}=\frac{\sin ^{2}(N \theta / 2)}{\sin ^{2}(q \theta / 2)}\left|\sum_{n=0}^{q-1} e^{-i n \theta} e^{i \phi_{n}}\right|^{2} .
$$

When $K$ is large that is when $q$ is much smaller than $N$, the fraction tends to a peaked function localized at values of $\theta$ given by $\theta_{\lambda}=2 \pi \lambda / q, \lambda$ integer. This behavior corresponds to the generation of $q$ optical pulses per period. In the time domain this corresponds to a repetition rate equal to $q f_{s}=p f_{c}$. The amplitude of the pulses is a priori modulated by the second term of the product: the peak intensity of the $\lambda^{\text {th }}$ pulse is given by:

$$
\begin{aligned}
I\left(\theta_{\lambda}\right) & =(N / q)^{2}\left|\sum_{n=0}^{q-1} e^{-i 2 \pi n \lambda / q} e^{i \phi_{n}}\right|^{2} \\
& =(N / q)^{2}\left|\sum_{n=0}^{q-1} e^{i \pi(n(n+1) p-2 \lambda n) / q}\right|^{2} .
\end{aligned}
$$

We obtain the expression of the square modulus of a Gauss sum that can be calculated relatively easily (see appendix A):

$$
I\left(\theta_{\lambda}\right)=N^{2} / q
$$

It is remarkable that the intensity of the $\lambda^{\text {th }}$ pulse does not depend on $\lambda$ : the resulting intensity consists in a set of $q$ pulses per period with identical amplitude (fig. 4).
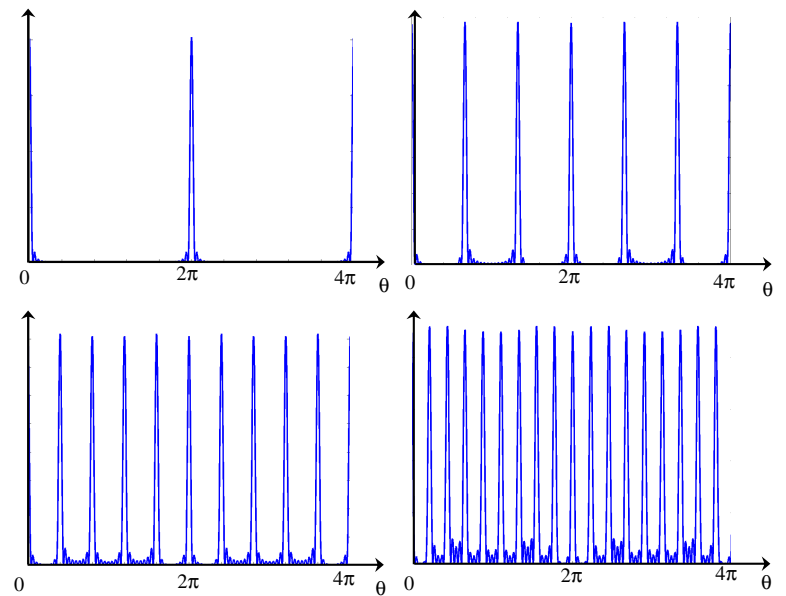

FIG. 4: Intensity of the FSF laser $I(\theta)$ computed with $N=45$ modes when $p / q=0$ (top left), $p / q=1 / 3$ (top right), $p / q=$ $1 / 5$ (bottom left) and $p / q=1 / 9$ (bottom right). A slight modulation of the peaks appears when the relation $q \ll N$ is no longer satisfied.

We now demonstrate that the width of the pulses when $q$ divides $N$ is Fourier transform-limited. The intensity is given by Eq. (9).

The first term is the familiar diffraction function of a grating of slits. The width of the pulses is equal to $2 \pi / K q=2 \pi / N$. The second term is a function defined as a Fourier series whose highest frequency is $q-1$, which means that the shortest variation scale of this term is $2 \pi /(q-1)$. Therefore this function can be considered as constant and equal to $q$ during a pulse, and the width of the pulse is therefore $2 \pi / N$. Note that the pulse width is limited only by the number of modes: the pulses generated by fractional Talbot effect are therefore Fourier transform-limited. We recover here the fact that the width of the diffraction fringes in fractional spatial Talbot effect is independent from the spatial frequency of the pattern. It is also noteworthy that the average intensity per period is equal to $<I>_{\theta}=1 / 2 \pi \times q \times I\left(\theta_{\lambda}\right) \times 2 \pi / N=$ $N$ which is consistent with the hypothesis of $N$ optical modes with amplitude unity.

\section{Case $q$ even and $p$ odd}

Similar conclusions can be obtained in the case where $q$ is even and $p$ is odd. We have then:

$$
\phi_{n+q}=\pi\left(n^{2}+2 n q+q^{2}+n+q\right) p / q=\phi_{n}+\pi .
$$

We also assume for simplicity reasons that $q$ divides $N$ $(N=K q)$. Then: 


$$
\begin{aligned}
F(\theta) & =\sum_{n=0}^{q-1} e^{-i n \theta} e^{i \phi_{n}} \sum_{k=0}^{K-1}(-1)^{k} e^{-i k q \theta} \\
& =\frac{1-e^{-i K(q \theta+\pi)}}{1-e^{-i(q \theta+\pi)}} \sum_{n=0}^{q-1} e^{-i n \theta} e^{i \phi_{n}}
\end{aligned}
$$

The intensity of the laser is therefore:

$$
I(\theta)=|F(\theta)|^{2}=\frac{\sin ^{2}(N(q \theta+\pi) / 2 q)}{\sin ^{2}((q \theta+\pi) / 2)}\left|\sum_{n=0}^{q-1} e^{-i n \theta} e^{i \phi_{n}}\right|^{2} .
$$

When $K$ is large that is when $q$ is much smaller than $N$, the fraction tends to a peaked function localized at values of $\theta$ given by $\theta_{\lambda}=\pi / q+2 \pi \lambda / q, \lambda$ integer. As before there are $q$ pulses per period $2 \pi$ and the repetition rate in the time domain in $q f_{s}$. Contrary to the previous case, the pulses are shifted from the origin of times by $\pi / q$.

The peak intensity of the $\lambda^{\text {th }}$ pulse is given by:

$$
\begin{aligned}
I\left(\theta_{\lambda}\right) & =(N / q)^{2}\left|\sum_{n=0}^{q-1} e^{-i(2 \pi \lambda+\pi) n / q} e^{i \phi_{n}}\right|^{2} \\
& =(N / q)^{2}\left|\sum_{n=0}^{q-1} e^{i \pi(n(n+1) p-n(2 \lambda+1)) / q}\right|^{2}
\end{aligned}
$$

A calculation similar to the previous case leads to:

$$
I\left(\theta_{\lambda}\right)=N^{2} / q
$$

which shows that the pulses have a constant amplitude (fig. 5).

The demonstration that the pulses are Fouriertransform-limited follows the same scheme as the case where $p$ is even or $q$ is odd.

\section{Remarks}

a. Case where $q$ does not divide $N$ In this case the amplitude of the peaks is modulated because of the remaining terms in Eq. (7). The modulation rate depends qualitatively on the distance between $N$ and the closest multiple of $q$. Fig. 6 shows that when $N$ is not a multiple of $q$, the amplitude of the pulses is not constant. For instance when $p / q=1 / 22$ only the two last terms of the sum in Eq. (6) are not taken in the sum $(200 \cong 2 \bmod 22)$ which results in relatively small amplitude fluctuations while in the case $p / q=1 / 23,7$ terms are not included in the sum $(200 \cong-7 \bmod 23)$ which results in larger amplitude fluctuations.
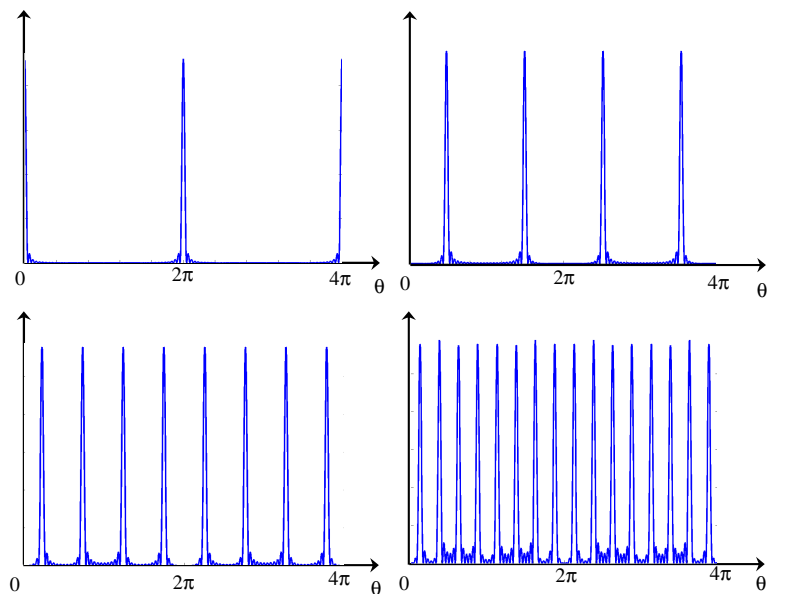

FIG. 5: Intensity of the FSF laser $I(\theta)$ computed with $N=$ 48 modes when $p / q=0$ (top left), $p / q=1 / 2$ (top right), $p / q=1 / 4$ (bottom left) and $p / q=1 / 8$ (bottom right).
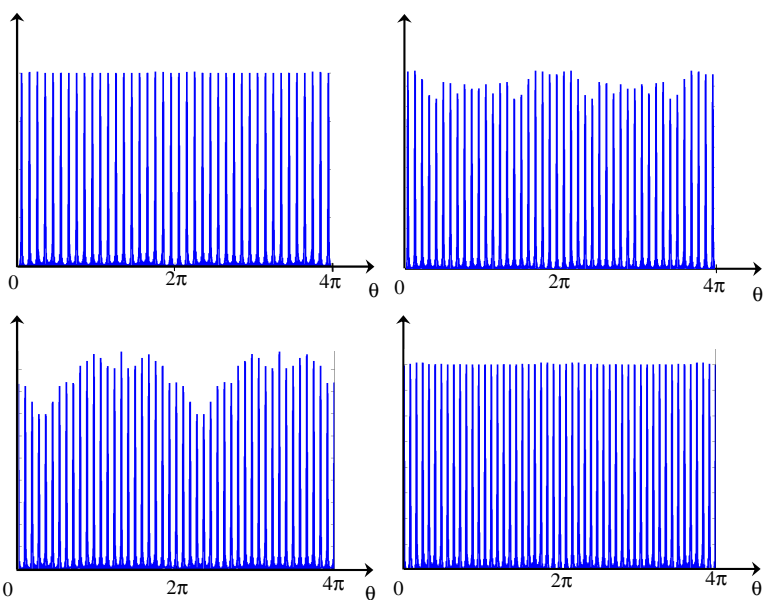

FIG. 6: Intensity of the FSF laser $I(\theta)$ computed with $N=$ 200 modes when $p / q=1 / 20$ (top left), $p / q=1 / 22$ (top right), $p / q=1 / 23$ (bottom left) and $p / q=1 / 25$ (bottom right).

b. Influence of the seed frequency The previous calculations involve the normalized time $\theta=\omega_{s} t-\omega_{0} \tau_{c}$. A notable influence of $\omega_{0}$ is to shift temporally the train of pulses. It can be of interest to describe the FSF laser as a system transforming a seed monochromatic field (i.e. a spectral Dirac) into a train of pulses (i.e. a temporal Dirac comb) with a temporal shift proportional to the seed frequency. The seeded FSF laser can be seen somehow as a temporal optical Fourier transform processor, where the spectrum of the seeding field is recorded in the temporal trace of the output of the laser. This could lead to applications in high speed signal processing using space-time duality [34]. 


\section{B. Phase and coherence of the pulses}

We now characterize the phase of the pulses, which corresponds to the phase of the complex numbers:

- $F\left(\theta_{\lambda}\right)=\frac{N}{q} \sum_{n=0}^{q-1} e^{-i 2 \pi \frac{n \lambda}{q}} e^{i \pi \frac{n p(n+1)}{q}}$ when $q$ is odd or $p$ is even

- $F\left(\theta_{\lambda}\right)=\frac{N}{q} \sum_{n=0}^{q-1} e^{-i \pi \frac{(2 n+1) \lambda}{q}} e^{i \pi \frac{n p(n+1)}{q}}$ when $q$ is even and $p$ is odd.

A complete determination of the phase of the Gauss sum arising in the expression of $F\left(\theta_{\lambda}\right)$ is cumbersome but can be found in $[4,35,36]$ :

- For $q$ odd and $p$ even:

$$
\begin{aligned}
& F\left(\theta_{\lambda}\right)=\frac{N}{\sqrt{q}}\left(\begin{array}{c}
p \\
q
\end{array}\right) \\
& \times \exp \left(-i \pi\left(\frac{q-1}{4}+\frac{p}{q}\left(\left[\frac{1}{p}\right]_{q}\right)^{2}\left(\frac{p+2 \lambda}{2}\right)^{2}\right)\right)
\end{aligned}
$$

- For $q$ odd and $p$ odd:

$$
\begin{aligned}
& F\left(\theta_{\lambda}\right)=\frac{N}{\sqrt{q}}\left(\begin{array}{c}
p \\
q
\end{array}\right) \\
& \times \exp \left(-i \pi\left(\frac{q-1}{4}+\frac{2 p}{q}\left[\frac{1}{2}\right]_{q}\left(\left[\frac{1}{2 p}\right]_{q}\right)^{2}(p+2 \lambda)^{2}\right)\right)
\end{aligned}
$$

- For $q$ even (and $p$ odd):

$$
\begin{aligned}
& F\left(\theta_{\lambda}\right)=\frac{N}{\sqrt{q}}\left(\begin{array}{c}
p \\
q
\end{array}\right) \\
& \times \exp \left(i \pi\left(\frac{p}{4}-\frac{p}{q}\left(\left[\frac{1}{p}\right]_{q}\right)^{2}\left(\frac{p+2 \lambda+1}{2}\right)^{2}\right)\right)
\end{aligned}
$$

where $\left[\frac{1}{a}\right]_{q}$ is the (unique) positive integer smaller than $q$ satisfying $a\left[\frac{1}{a}\right]_{q}=1 \bmod q .\left(\begin{array}{c}a \\ b\end{array}\right)$ is the Jacobi symbol, equal to +1 if there is an integer $m$ such that $m^{2} \equiv a[b]$ and -1 otherwise. The resulting phases are plotted on fig. 7 .

\section{Pulse to pulse coherence}

We determine the coherence properties between two pulses at times $\theta_{\lambda}$ and $\theta_{\lambda+\mu}$ by calculating $F^{*}\left(2 \pi \frac{\lambda+\mu}{q}\right) F\left(2 \pi \frac{\lambda}{q}\right)$. (We assume here that $p$ is even or

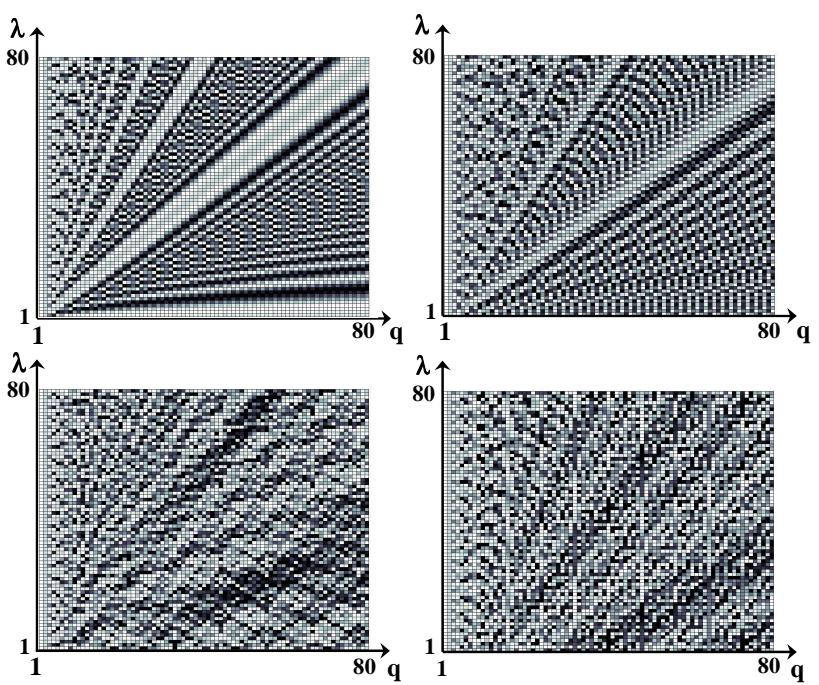

FIG. 7: Two-dimensional plot of $\cos \left[\arg \left(F\left(\theta_{\lambda}\right)\right)\right]$ as a function of $q$ and $\lambda$, when $p=1$ (top left), $p=2$ (top right), $p=7$ (bottom left) and $p=11$ (bottom right). The grey scale is adjusted between black $(-1)$ and white $(+1)$.

$q$ is odd but the other case would be treated similarly.) It can be shown (see appendix B) that:

$$
\left\langle F^{*}\left(2 \pi \frac{\lambda+\mu}{q}\right) F\left(2 \pi \frac{\lambda}{q}\right)\right\rangle_{\lambda}=\frac{N^{2}}{q} \delta(\mu \bmod q)
$$

where $\delta(\mu \bmod q)=1$ when $\mu$ divides $q$ and 0 else. $<>_{\lambda}$ denotes the average over $\lambda$.

The interference term between two pulses separated by $2 \pi \mu / q$ vanishes in average when $\mu$ is not a multiple of $q$. Note that this constitutes a substantial difference with mode-locked lasers where all pulses show the same phase. This property accounts for the fact that when an interferometric autocorrelation is performed by second harmonic generation of the output of the CW-seeded FSF laser, the satellite peaks (arising from the correlation of two different pulses) show no interference fringes, contrary to the central autocorrelation trace [32].

\section{Intensity spectrum}

We now turn to the intensity spectrum of the CWseeded FSF laser by deriving an expression if $I(\theta)$ as a Fourier series. Starting from:

$$
I(\theta)=|F(\theta)|^{2}=\sum_{n, m=0}^{N-1} e^{-i(n-m) \theta} e^{i \frac{n(n+1)-m(m+1)}{2} \phi}
$$

we divide the double sums into three terms, according to: 


$$
\sum_{n, m=0}^{N-1} A_{n, m}=\sum_{n=0}^{N-1} A_{n, n}+\sum_{l=1}^{N-1} \sum_{n=l}^{N-1} A_{n, n-l}+\sum_{l=1}^{N-1} \sum_{n=l}^{N-1} A_{n-l, n} .
$$

The first term is equal to $N$. The third term is simply the complex conjugate of the second one and:

$$
\begin{aligned}
I(\theta) & =N+2 \Re\left(\sum_{l=1}^{N-1} \sum_{n=l}^{N-1} e^{-i l \theta} e^{i \frac{l(2 n-l)}{2} \phi}\right) \\
& =N+2 \Re\left(\sum_{l=1}^{N-1} e^{-i l \theta} e^{i \frac{l(l+1)}{2} \phi} \frac{1-e^{i l(N-l) \phi}}{1-e^{i l \phi}}\right) .
\end{aligned}
$$

Assuming $\phi=2 \pi p / q$,

$$
I(\theta)=N+2 \Re\left(\sum_{l=1}^{N-1} e^{-i l \theta} e^{i \pi \frac{p l(l+1)}{q}} \frac{1-e^{i 2 \pi \frac{l p(N-l)}{q}}}{1-e^{i 2 \pi \frac{l p}{q}}}\right) .
$$

The intensity is expressed as a Fourier series whose coefficient of order $l$ is proportional to $\frac{1-e^{i 2 \pi \frac{l p(N-l)}{q}}}{1-e^{i 2 \pi \frac{l p}{q}}}$. Provided $N$ is large enough compared to $q$ this term vanishes except when $l$ is a multiple of $q$. Defining $l=s q$, with $s$ integer leads to:

$$
I(\theta)=N+2 \Re\left(\sum_{s=1}^{E\left(\frac{N-1}{q}\right)}(N-s q) e^{-i s q \theta} e^{i \pi p s(s q+1)}\right)
$$

where $E(x)$ is the integer part of the real number $x$. Finally,

$$
I(\theta)=N+2 \sum_{s=1}^{E\left(\frac{N-1}{q}\right)}(N-s q) \cos (s q \theta-\pi p s(s q+1)) .
$$

It is noteworthy that contrary to the optical spectrum, which consists in a comb with a frequency spacing equal to $f_{s}$, the intensity spectrum shows a frequency spacing equal to $q f_{s}$ (fig. 8): the optical field is $2 \pi$ periodic while the intensity is $2 \pi / q$ periodic. The corresponding decimation in the spectral components results from the interference of waves with quadratic phases. Actually the difference between the optical and the intensity spectrum is a general feature of FSF lasers [37]. Recall that in the case where the FSF laser is injected with spontaneous emission (modeless laser), the same kind of behavior is observed: the optical spectrum is continuous while the intensity spectrum is discrete [38]. The counterpart in the temporal domain temporal is the fact that the degree of first order coherence is peaked around null delay while the degree of second order coherence is periodic in modeless lasers [39].
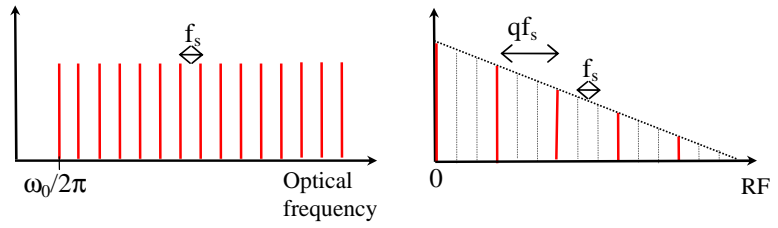

FIG. 8: Left: optical spectrum. Right: corresponding intensity spectrum when $q=3$. Note the decimation in the frequencies.

\section{E. Intensity fluctuations}

We now turn to the dependence of the intensity fluctuations of the laser with $\phi$. This constitutes a pertinent parameter to study the time properties of seeded FSF lasers, since it enables to characterize the pulsing regime and the repetition rate of the laser. Moreover the intensity fluctuations can be measured experimentally with second harmonic generation (SHG). We define the relative fluctuations of the intensity as $W(\phi)=<$ $I(\theta)^{2}>_{\theta} /<I(\theta)>_{\theta}^{2}(\phi)$ where $<>_{\theta}$ denotes the average over time $(\theta)$. Recall that when $\phi=2 \pi p / q$ with $\mathrm{q}$ much smaller that N, the laser output consists in Fourier transform-limited pulses with a repetition rate equal to $\mathrm{q}$. The peak intensity of the pulses is $N^{2} / q$ and the pulse duration is $2 \pi / N$. Therefore $<I(\theta)^{2}>_{\theta} \sim N^{3} / q$ and $<I(\theta)>_{\theta}^{2} \sim N^{2}$ which leads to a rough approximation of $W(\phi):$

$$
W(\phi=2 \pi p / q)=\frac{<I(\theta)^{2}>_{\theta}}{<I(\theta)>_{\theta}^{2}}(\phi=2 \pi p / q) \sim N / q .
$$

When $\mathrm{N}$ increases, this function tends to the so-called self-similar Thomae's function defined by function defined on $[0,1]$ by: $T(p / q)=1 / q$ when $p$ and $q$ are coprimes, and $T(\eta)=0$ when $\eta$ is irrational

A more rigorous expression of the intensity fluctuations can be derived using the expression of the intensity as a Fourier series. Starting from Eq. (27) one has $<I(\theta)>_{\theta}^{2}=N^{2}$ and:

$$
<I(\theta)^{2}>(\phi)=N^{2}+2 \sum_{l=1}^{N-1} \frac{\sin ^{2}(l(N-l) \phi / 2)}{\sin ^{2}(l \phi / 2)} .
$$

Therefore:

$$
W(2 \pi p / q)=1+\frac{2}{N^{2}} \sum_{l=1}^{N-1} \frac{\sin ^{2}(\pi l(N-l) p / q)}{\sin ^{2}(\pi l p / q)} .
$$

A significant contribution to the sum arises only from indexes $l$ satisfying $l p / q$ integer. Since $p$ and $q$ are coprimes, $l$ is then a multiple of $q$, i.e. $l=k q$. Then: 


$$
W(2 \pi p / q)=1+\frac{2}{N^{2}} \sum_{k=1}^{E\left(\frac{N-1}{q}\right)}(N-k q)^{2} .
$$

In the case where $q$ is much smaller than $N$, $\sum_{k=1}^{E\left(\frac{N-1}{q}\right)}(N-k q)^{2} \simeq(1 / q) \int_{0}^{N} x^{2} d x=N^{3} / 3 q$.

Therefore we obtain:

$$
W(2 \pi p / q)=1+\frac{2}{3} \frac{N}{q} .
$$

and we recover the previous link to the Thomae's function as in Eq. (30). Note that this expression is valid in the limit where $q$ is much smaller that $N$. This expression shows a good agreement with the computed intensity fluctuations plotted on fig. 9. Note the agreement with the experimental variation of the intensity fluctuations measured by SHG and reported in [32]

Finally an interesting situation occurs when $p=1$ and $q=N$. In this case, we have:

$$
W(2 \pi / N)=1+\frac{2}{N^{2}} \sum_{l=1}^{N-1} \frac{\sin ^{2}(\pi l(N-l) / N)}{\sin ^{2}(\pi l / N)} .
$$

It can be proven that the sum in this expression scales as $N^{3 / 2}$ [40], which ensures that $W(2 \pi / N)$ tends to 1 when $N$ is large. In this case, the relative intensity fluctuations tend to vanish. Note that by tuning $\phi$ between 0 and $2 \pi / N$, and with a constant spectral content, the FSF laser can be used as a source of intensity fluctuations tunable continuously between $1+2 N / 3$ and 1 . In fact $W$ can be identified as $g^{2}(\tau=0)$, that is the degree of second order coherence at zero delay. Recall that a chaotic source (resp. a single mode laser) shows $g^{2}(0)=2$ (resp. 1) [41]. A source with tunable intensity fluctuations could find applications in the field of quantum imaging [42], while the possibility to lower the intensity fluctuations of a broadband laser could be used to cancel deleterious non-linear effects. Finally it is interesting to reconsider the equivalent of this temporal effect, in the spatial domain. The spatial equivalent of a comb of optical modes showing a constant intensity would consist in a grating with isotropic diffraction or constant albedo. It is remarkable that this concept has been developed for years in acoustics [43] where quadratic residue diffusers enable isotropic scattering of sound and applied in the design of concert halls $[44,45]$. Similarly in the optical domain, the use of quadratic residue diffusers could be applied as a technique of speckle suppression in light projection devices.

\section{ARBITRARY AMPLITUDE OF THE OPTICAL MODES}

We now provide a more realistic description of a seeded FSF laser by taking into account the spectral properties
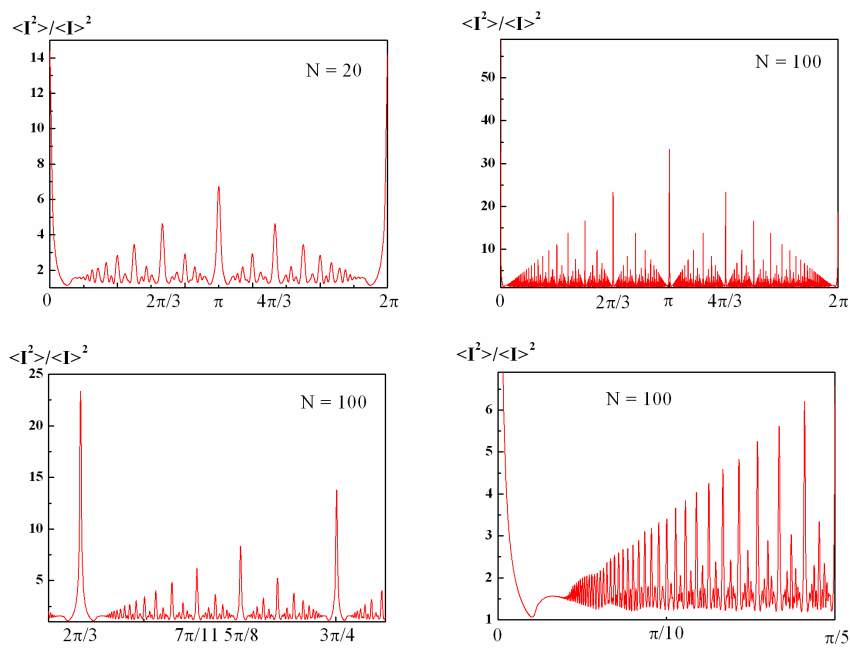

FIG. 9: Intensity fluctuations $W(\phi)=\frac{\left\langle I(\theta)^{2}\right\rangle_{\theta}}{\langle I(\theta)\rangle_{\theta}^{2}}(\phi)$ computed when $N=20$ (top left) and 100 (top right). Bottom left (resp. right): zoom around $\phi=5 \pi / 8$ (resp. $\phi=0$ ) for $N=100$. Note the dip in the intensity fluctuations when $\phi=2 \pi / N=\pi / 50$.

of the laser as defined in the first section. In fact it turns out that the results are basically unchanged, which corresponds to the fact in the spatial domain, that when shining a grating of slits, the diffraction pattern does not critically depend on the amplitudes of the secondary sources or equivalently, on the intensity of the light incident at each slit. Moreover it turns out that a smooth spectral shape results in a suppression in the modulation of the amplitude of the pulses observed on fig. 6 .

\section{A. Electric field at the output of a FSF laser}

We consider the general expression for the electric field at the output of the CW injection-seeded FSF laser:

$$
E(t)=E_{0} e^{-i \omega_{0} t} \sum_{n} g(n) e^{-i n \omega_{s} t} e^{i n \omega_{0} \tau_{c}} e^{i \frac{n(n+1)}{2} \phi}
$$

where $\phi=2 \pi f_{s} \tau_{c}=2 \pi f_{s} / f_{c}$. The resulting field is therefore a defined by a theta series consisting of a sum of phasors with non-constant amplitudes and quadratic phases. The corresponding plot in the complex plane consists therefore in a succession of Cornu spirals with variable sizes, in contrast to the previous case (fig. 10).

\section{B. Intensity}

The intensity of the laser field is $I(t)=E(t) E^{*}(t)$. Writing $I_{0}=\left|E_{0}\right|^{2}$, one has: 

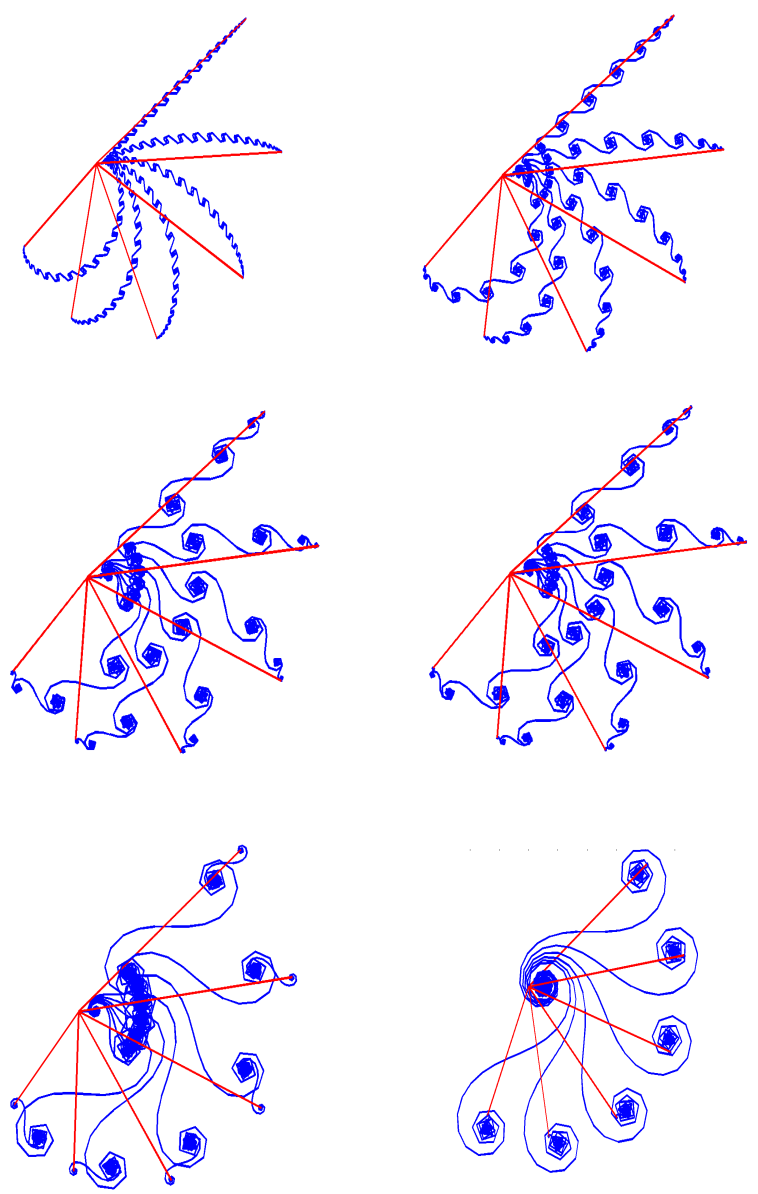

FIG. 10: Representation in the complex plane of $F(\theta)$ as a sum of $N=200$ phasors with the gaussian envelope defined on fig. 11 and quadratic phases. The resulting field corresponds to the straight line. Six consecutive values of $\theta$ are considered: $\theta=2 k \pi / 1000,0 \leq k \leq 5$. The resulting field evolves clockwise. For all cases $p=1$, and $q=7,15,25,27,51,89$ from left to right and top to bottom.

$I(t)=I_{0} \sum_{n, m} g(n+m) g(n) e^{-i m\left(\omega_{s} t-\omega_{0} \tau_{c}\right)} e^{i n m \phi} e^{i \frac{m(m+1)}{2} \phi}$.

The resulting intensity in the case of a Gaussian spectrum is plotted on fig. 11. Note that the amplitude of the pulses show no temporal modulation for all values of $q$, contrary to fig. 6 .

The Poisson summation formula applied to the sum over $n$ yields:

$$
I(t)=I_{0} \sum_{n, m} G(m, n-m \phi / 2 \pi) e^{-i m\left(\omega_{s} t-\omega_{0} \tau_{c}\right)} e^{i \frac{m(m+1)}{2} \phi}
$$

where $G\left(x_{1}, x_{2}\right)=\int g\left(y+x_{1}\right) g(y) e^{-2 i \pi y x_{2}} d y$. In the two-dimensional plane, $G\left(x_{1}, x_{2}\right)$ is a function localized

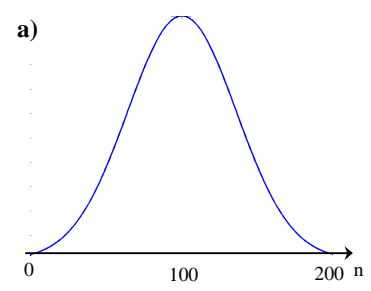

b)
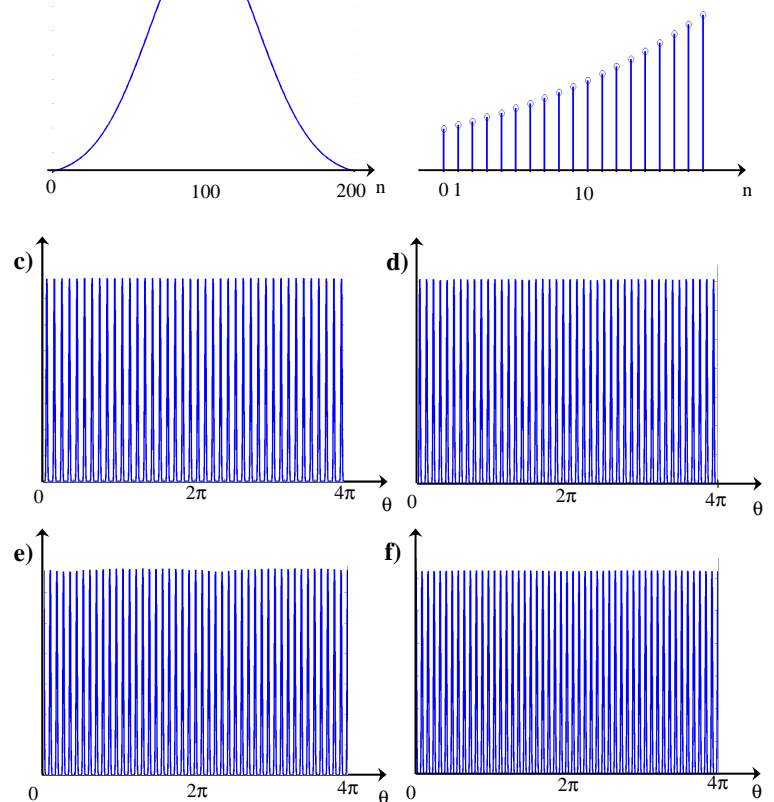

FIG. 11: a) Envelope of the (gaussian) spectrum of the FSF laser. b) Zoom on the first modes. Bottom: intensity of the FSF laser $I(\theta)$ computed with the previous gaussian spectrum when $p / q=1 / 20$ (c), $p / q=1 / 22$ (d), $p / q=1 / 23$ (e) and $p / q=1 / 25$ (f). Contrary to the case where all modes have the same amplitude, the pulse-to-pulse fluctuations tend to disappear.

at the origin. It is important to observe that the width of $G$ along the first coordinate evolves proportionally to $N$, the cut-off limit of $g$ while the width along the second coordinate scales as $1 / N$. Therefore only integers $n$ and $m$ satisfying $m<N$ and $n-m \phi / 2 \pi<1 / N$ contribute to the intensity. It is particularly interesting to consider the case $\phi=2 \pi p / q$ where $p$ and $q$ are coprime integers and $q<N$. In this specific case the main contribution arises from integers $n$ and $m$ satisfying $n / m=p / q$ (i.e. $m=k q$ and $n=k p, k$ integer) and the intensity rewrites:

$$
I(t)=I_{0} \sum_{k} G(k q, 0) e^{-i k q\left(\omega_{s} t-\omega_{0} \tau_{c}\right)} e^{i \pi p k(k q+1)} .
$$

One assumes for simplicity that $p$ is even (the case of odd $p$ can be treated similarly). Then $I(t)=$ $I_{0} \sum_{k} G(k q, 0) e^{i k q\left(\omega_{s} t+\omega_{0} \tau_{c}\right)}$. Since $G(k q, 0)$ is real this Fourier series corresponds to pulses at the repetition rate $q f_{s}=p f_{c}$, according to $\phi=2 \pi f_{s} / f_{c}=2 \pi p / q[31]$. We recover here a repetition rate multiple of the frequency spacing $f_{s}$ due to the temporal fractional Talbot effect. 


\section{Temporal width of the pulses}

It is also instructive to derive the temporal width of the light pulses when $\phi=2 \pi p / q$. The intensity is maximum at time $t_{m}=-\omega_{0} \tau_{c} / \omega_{s}$. We assume again that $p$ is even but the other case would be treated similarly. Then $I\left(t_{m}\right)=I_{0} \sum_{k} G(k q, 0)$. Defining $\tau$ so that $I\left(t_{m}+\tau / 2\right)=$ $I\left(t_{m}\right) / 2$ a Taylor expansion of $I(t)$ around $t_{m}$ leads to:

$$
\left(\omega_{s} \tau / 2\right)^{2} \approx \frac{\sum_{k} G(k q, 0)}{\sum_{k}(k q)^{2} G(k q, 0)} .
$$

Provided $N \gg 1$ that is $\omega_{s} \ll \Delta \omega$, we have $\left(\omega_{s} \tau / 2\right)^{2} \approx$ $\int G(x, 0) d x / \int G(x, 0) x^{2} d x \approx\left(\omega_{s} / \Delta \omega\right)^{2}=1 / N^{2}$. Therefore the pulse duration when $\phi$ is a rational multiple of $2 \pi$, is independent from $q$ and also inversely proportional to the spectral width of the laser. We conclude that the pulses emitted by the seeded FSF laser are Fourier transform-limited and independent from the repetition rate.

\section{Intensity spectrum}

In the spectral domain, going back to the general case and defining $\tilde{I}(\Omega)=\int I(t) e^{-i \Omega t} d t$, Eq. (38) leads to:

$\tilde{I}(\Omega)=I_{0}\left[\sum_{m} \delta\left(\Omega-m \omega_{s}\right) e^{i \psi_{m}}\right]\left[\sum_{n} G\left(\Omega / \omega_{s}, n-\Omega / \omega_{c}\right)\right]$

where $\psi_{m}=m \omega_{0} \tau_{c}+\pi(m+1) \Omega / \omega_{c}$.

The intensity spectrum of the seeded FSF laser appears as the product of a frequency comb with a spacing of $\omega_{s}$ by a second comb of peaks separated by $\omega_{c}$. The width of the low-frequency modes of the second comb can be estimated by using the fact that when $\Omega \ll \Delta \omega$, $\Omega / \omega_{s} \ll N$ and then $G\left(\Omega / \omega_{s}, n-\Omega / \omega_{c}\right) \simeq G(0, n-$ $\left.\Omega / \omega_{c}\right)$. Therefore the width of the peaks is $\delta \Omega=\omega_{c} / N=$ $\omega_{c} \omega_{s} / \Delta \omega$. Interestingly, $\delta \Omega$ can be interpreted as the inverse of the photon cavity lifetime.

\section{E. Intensity fluctuations}

One now focuses on the intensity fluctuations in the general case (i.e. $p$ can be odd or even). One has:

$$
W(\phi)=\sum_{n, m, l} G(m, n-m \phi / 2 \pi) G^{*}(m, n-m \phi / 2 \pi+l) .
$$

The leading contribution comes from $l=0$. Then $W(\phi)=\sum_{n, m}|G(m, n-m \phi / 2 \pi)|^{2}$ which shows maxima when $\phi=2 \pi p / q$ according to Eq. (38). In the neighborhood of each maximum we set $\phi=2 \pi(p / q+\epsilon)$ and obtain in the limit $\omega_{s} \ll \Delta \omega$ and $\epsilon$ small, the continuum approximation:

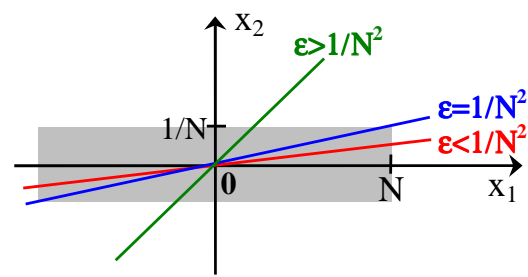

FIG. 12: Interpretation of the width of the peaks in the intensity fluctuations. The grey rectangle represents the function $\left|G\left(x_{1}, x_{2}\right)\right|^{2}$. The intensity fluctuations at $\phi=2 \pi(p / q+\epsilon)$ are given by the integration of $\left|G\left(x_{1}, x_{2}\right)\right|^{2}$ performed along the lines $x_{2}=\epsilon x_{1}$. When $\epsilon>1 / N^{2}$, the integral - and consequently the intensity fluctuations - decrease.

$$
W(2 \pi(p / q+\epsilon)) \approx \frac{1}{q} \int|G(x, \epsilon x)|^{2} d x .
$$

The intensity fluctuations when $\phi=2 \pi p / q$ are therefore proportional to $1 / q$, and we recover the generic self-similar Thomae's function. When $N=\Delta \omega / \omega_{s}$ increases, the function $\int|G(x, \epsilon x)|^{2} d x$ becomes sharply peaked around $\epsilon=0$. This result is in full agreement with the experimental results reported in [32] where the peaks in the plot of the intensity fluctuations measured by SHG get narrower when the spectral bandwidth of the laser (i.e. the total number of modes) is increased.

\section{PERSPECTIVES AND LIMITATIONS}

\section{A. Generation of ultra-high repetition rates}

CW-seeded FSF lasers are a promising solution to generate Fourier transform limited pulses with tunable and possibly ultrahigh repetition rates (in fact limited only by the spectral bandwidth of the laser). We now address the problem of the stability of the repetition rate of the pulse train when $\phi$ is set close to $2 \pi p / q$. Then the repetition rate is equal to $q f_{s}$, that is a multiple of a $\mathrm{RF}$ frequency. We assume that the latter can be driven by a high precision $\mathrm{RF}$ clock and we evaluate the requirement on the stabilization of the cavity length to maintain this repetition rate. The question is how precisely the cavity must be controlled to generate Fourier-transform limited pulses at repetition rate $q f_{s}$. A convenient criteria is given by the width of the intensity fluctuations $W(\phi)$ in the vicinity of $\phi=2 \pi p / q$.

Starting from Eq. (43), it can be intuitively shown (fig. 12 ) that the cutoff value corresponds to $\epsilon_{\text {cut off }}=1 / N^{2}$. Therefore the requirement on the precision of $\phi$ is $\delta \phi=$ $2 \pi / N^{2}$ : a plot of the intensity as a function of $\phi$ in the vicinity of $2 \pi p / q$ is given on fig. 13 and confirms that within a range equal to $1 / N^{2}$ around $p / q$, the repetition 

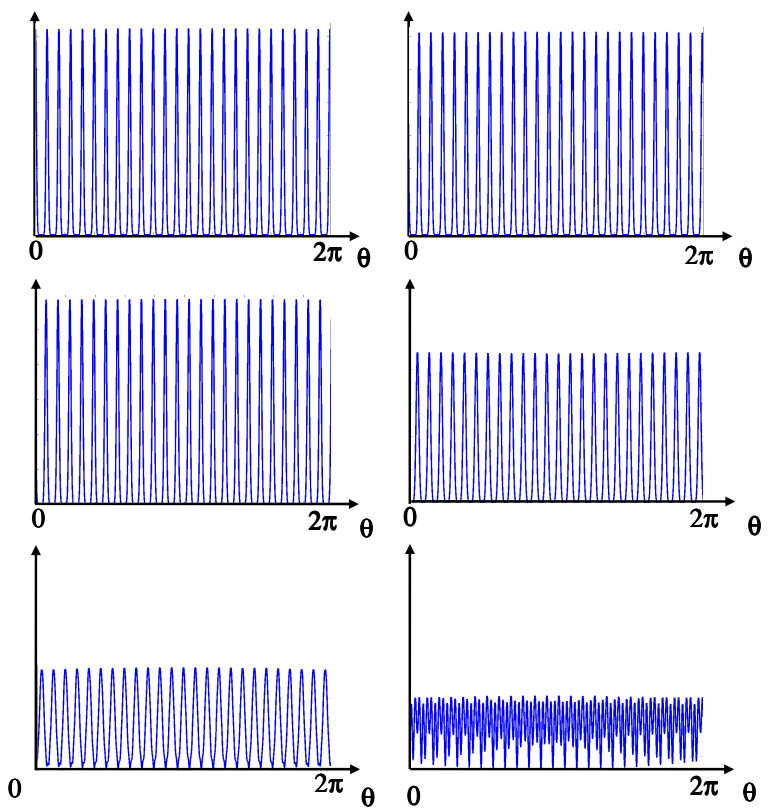

FIG. 13: Intensity of the FSF laser $I(\theta)$ computed with the gaussian spectrum defined on fig. 11 for different values of $\phi$ in the vicinity of $2 \pi(1 / 25)$. The vertical scale is unchanged. From left to right and top to bottom: $\phi=$ $2 \pi(1 / 25), 2 \pi\left(1 / 25+1 / 200^{2}\right), 2 \pi\left(1 / 25+2 / 200^{2}\right), 2 \pi(1 / 25+$ $\left.5 / 200^{2}\right), 2 \pi\left(1 / 25+10 / 200^{2}\right), 2 \pi\left(1 / 25+20 / 200^{2}\right)$.

range is unchanged. The corresponding requirement on the cavity length, that is on $\omega_{c}$ is $\delta \omega_{c} / \omega_{c}=\omega_{c} /\left(N^{2} \omega_{s}\right)$. This requirement was not critical yet in the experiment we described [32], but becomes challenging for THz rates: typically for a spectral bandwidth of $10 \mathrm{THz}$, a cavity free spectral range and a frequency shift per roundtrip both close to $300 \mathrm{MHz}$, a repetition rate of $1 \mathrm{THz}$ (i.e. $q \approx 3000$ ) the cavity needs to be stabilized at the scale of $10 \mathrm{~nm}$. It is instructive to compare this requirement with the case of a mode-locked laser whose repetition rate is fixed by the cavity length. In the case of a CW injectionseeded FSF laser, provided the cavity free spectral range is stabilized within $\delta \omega_{c}=\omega_{c}^{2} /\left(N^{2} \omega_{s}\right)$, the pulses can show some distortion but the repetition rate is unchanged (fig. 13). In the case of a modelocked laser, the repetition rate would change by $\delta \omega_{c} / 2 \pi$. Finally another advantage of CW-seeded FSF lasers for stable high repetition rates is the possibility to lock the cavity length simply to the local maximum of the intensity fluctuations $W(\phi)$. The latter is easily accessible experimentally since it is proportional to the second harmonic generation (SHG) signal: the cavity stabilization does not require a comparison with an external RF clock signal as in modelocked lasers.

\section{B. Jitter and linewidth of the seed laser}

As previously mentioned, the frequency stability of the seed laser plays a determining role on the spectral purity of the repetition rate of the pulse train. From Eq. (2) it is clear that a frequency jitter of $\delta \omega_{0}$ induces a time jitter equal to $\delta t=\frac{\delta \omega_{0}}{\omega} \tau_{c}$. Note that this expression is valid in the case where the frequency jitter of the seed laser occurs on a time scale larger than the photon cavity lifetime in the cavity, that is to say $N \tau_{c}$. For faster fluctuations, the timing jitter is accompanied by a temporal broadening of the pulses. Therefore a special care must be dedicated to the spectral properties of the seed laser. A standard ECDL (extended cavity diode laser) has a linewidth of about $100 \mathrm{kHz}$, which results in a typical time jitter of 3 ps. To reach time jitter below $10 \mathrm{fs}$ as required in optical sampling systems $[18,46]$, a seed linewidth no larger than a few $\mathrm{kHz}$ is needed, which can be achieved by distributed feedback fiber lasers [47]. Finally the stabilization of the seed laser to a reference molecular absorbtion line or to a ultrastable optical cavity can lower significantly the time jitter of the train of pulses at the output of the FSF laser. Therefore a FSF laser seeded with a singlemode laser is expected to be a robust source of highfidelity clock signals and shows promising perspectives for optoelectronic applications like optical sampling of electric signals.

\section{Dispersion and non-linear effects}

Finally to ensure Fourier transform-limited pulses in broadband ultra-high repetition rate FSF lasers, intracavity dispersion becomes crucial. Consider in the cavity a medium of length $L$ showing both first and second order dispersion terms. The wave vector is $k(\omega)=$ $k_{0}+\alpha\left(\omega-\omega_{0}\right)+\beta / 2\left(\omega-\omega_{0}\right)^{2}$ where $k_{0}=k\left(\omega_{0}\right)$, $\alpha=(\partial k / \partial \omega)\left(\omega_{0}\right)$ and $\beta=\left(\partial^{2} k / \partial \omega^{2}\right)\left(\omega_{0}\right)$. The additional phase shift acquired during roundtrip $n$ is

$$
\begin{aligned}
& \phi_{n}-\phi_{n-1}=\left(\omega_{0}+n \omega_{s}\right) \tau_{c}+k\left(\omega_{0}+n \omega_{s}\right) L \\
= & \omega_{0} \tau_{c}+k_{0} L+n\left(\omega_{s} \tau_{c}+\alpha \omega_{s} L\right)+n^{2} \frac{\beta \omega_{s}^{2} L}{2} .
\end{aligned}
$$

Finally the phase of mode $n$ is:

$$
\begin{aligned}
\phi_{n} & =n\left(\omega_{0} \tau_{c}+k_{0} L\right)+\frac{n(n+1)}{2}\left(\omega_{s} \tau_{c}+\alpha \omega_{s} L\right) \\
& +\frac{n(n+1)(2 n+1)}{12} \beta \omega_{s}^{2} L .
\end{aligned}
$$

The first order dispersion $\alpha$ results in an additional contribution to the quadratic phase and can therefore be compensated by tuning the cavity length or changing the frequency of the AOFS. On the contrary the group velocity dispersion $\beta$ leads to the appearance in the phase shift of a cubic term, which is expected to have a strong 
influence on the output laser field. However standard techniques of cancelation of group velocity dispersion should enable a precise control of this deleterious effect, to recover Fourier-transform limited pulses. Finally it is worth pointing out that possible non-linear effects in the laser cavity (Kerr effect, self-phase modulation) have a limited impact in high repetition rate FSF lasers: indeed the energy is spread over $p$ pulses per roundtrip and third order non-linear effects are accordingly divided by $p^{3}$.

\section{Conclusion}

We have given a comprehensive theoretical description of Talbot lasers, i.e. CW injection-seeded FSF lasers, sufficient to account for all experimental results published in [32]. We provided a simple model of a Talbot laser, consisting in a set of optical modes separated by a constant frequency spacing $f_{s}$ and exhibiting quadratic phases defined by $\phi_{n}=n(n+1) \phi / 2$. The resulting properties have been related to the fractional Talbot effect in optical diffraction and are connected to number theory by the appearance of Gauss sums and theta series. First we detailed the case where $N$ equidistant optical modes have a constant amplitude. We gave a description of the electric field, of the intensity in the case where $\phi$ is a rational multiple of $2 \pi$ : when $\phi=2 \pi p / q$, with $q<N$ the resulting field consists in a train of regular light pulses at the repetition rate equal to $q f_{s}$. The pulses are shown to be Fourier transform-limited and the phases of the pulses are uncorrelated on average. We characterized the intensity fluctuations of the resulting light field and proved among others, that Talbot lasers can also be used as a broadband light source with tunable intensity fluctuations. Then we considered a more realistic description of a CW-seeded FSF laser by taking into account the spectral shape of the resulting field. In this case we have shown a cancelation of the pulse-to-pulse fluctuations, while results similar to the previous case have been obtained.

This work is expected to have significant outcomes in the domain of high repetition rate lasers. On the basis of the experimental results obtained in [32], we anticipate that much higher values of the repetition rate can be obtained: a recent demonstration of a seeded Ti:Sa FSF laser with a spectral bandwidth as large as a few nm [47] leads to the possibility to generate lasers with $\mathrm{THz}$ repetition rates, which can be used for the generation of $\mathrm{THz}$ waves, for ultrafast digital communications systems, for the time transfer of remote clocks and ultrafast optical cadencers for analog-to-digital converters, or for the optical sampling of microwave signals. Another advantage of this source in opto-electronics relies on the fact that contrary to conventional mode-locked lasers where the repetition rate is set by the cavity length, the stability of the repetition rate in CW injection-seeded FSF lasers is directly linked to the stability of the shift frequency which can be controlled with an extremely high precision through standard quartz or atomic clocks. Finally we also expect Talbot lasers to find applications in spec- troscopy, by enabling the resonant excitation of acoustic modes of metallic nanoparticles [19, 20].

This work was supported in part by the CNRS and the LIPhy. We warmly thank J. Marklof for his stimulating interest in the subject, and H. Cohen for his help on Gauss sums. We also acknowledge A . Garnache and Y. Colin de Verdière for fruitful discussions.

\section{APPENDIX}

\section{A. Calculation of the amplitude of the pulses}

We consider the case where $q$ is odd or $p$ is even (the other case would be treated similarly). The amplitude of the pulse $\# \lambda$ is given by $F\left(\theta_{\lambda}\right)=$ $(N / q) \sum_{n=0}^{q-1} e^{i \pi(n(n+1) p-2 \lambda n) / q}$. Note that $F\left(\theta_{\lambda+q}\right)=$ $F\left(\theta_{\lambda}\right)$, which shows that the field reproduces itself after $q$ pulses, a direct consequence of the fact $F(\theta)$ is $2 \pi$ periodic. It can be shown that changing $n$ into $n+q$ leaves the sum invariant. Then:

$$
F\left(\theta_{\lambda}\right)=\frac{N}{q} \sum_{n \bmod q} e^{i \pi(n(n+1) p-2 \lambda n) / q}
$$

where the summation modulo $q$ can be performed over any set of $q$ consecutive integers. The square modulus is given by:

$$
\left|F\left(\theta_{\lambda}\right)\right|^{2}=\frac{N^{2}}{q^{2}} \sum_{m, n \bmod q} e^{i \pi\left(\left(n^{2}-m^{2}\right) p+(n-m)(p-2 \lambda)\right) / q}
$$

Writing $l=n-m$ leads to:

$$
\begin{aligned}
\left|F\left(\theta_{\lambda}\right)\right|^{2} & =\frac{N^{2}}{q^{2}} \sum_{l, m \bmod q} e^{i \pi l((l+2 m+1) p-2 \lambda) / q} \\
& =\frac{N^{2}}{q^{2}} \sum_{l \bmod q} e^{i \pi l((l+1) p-2 \lambda) / q} \sum_{m \bmod q} e^{i 2 \pi l m p / q}
\end{aligned}
$$

The second term of the product vanishes except when $q$ divides $l p$. Since $p$ and $q$ are coprimes, $l$ must be a multiple of $q$ chosen modulo $q$. Therefore $l=0$ which leads to $I\left(\theta_{\lambda}\right)=\left|F\left(\theta_{\lambda}\right)\right|^{2}=N^{2} / q$.

\section{B. Pulse-to-pulse coherence}

We consider the case where $q$ is odd or $p$ is even (the other case would be treated similarly). The interference term between two pulses at times $\theta_{\lambda+\mu}=2 \pi \frac{\lambda+\mu}{q}$ and $\theta_{\lambda}=2 \pi \frac{\lambda}{q}$ is: 
The average over q pulses is:

$$
\begin{aligned}
& F^{*}\left(\theta_{\lambda+\mu}\right) F\left(\theta_{\lambda}\right) \\
= & \frac{N^{2}}{q^{2}} \sum_{n, m \bmod q} e^{i \pi\left(\left(n^{2}-m^{2}\right) p+(n-m)(p-2 \lambda)+2 m \mu\right) / q} \\
= & \frac{N^{2}}{q^{2}} \sum_{l \bmod q} e^{i \pi \frac{l^{2} p+l(p-2 \lambda)}{q}} \sum_{m \bmod q} e^{i 2 \pi \frac{m(\mu+l p)}{q}} \cdot
\end{aligned}
$$

If $\mu$ is a multiple of $q$, the second sum vanishes excepted when $l=0$. In this case: $F^{*}\left(\theta_{\lambda+\mu}\right) F\left(\theta_{\lambda}\right)=N^{2} / q$ and the phases of the pulses are identical: we recover here the fact that $F\left(\theta_{\lambda}\right)=F\left(\theta_{\lambda+\mu}\right)$.

If $q$ does not divide $\mu$ the second sum does not vanish only when $l p+\mu$ is a multiple of $q$. Then there is an integer $l_{0}$ satisfying $l p=s q-\mu$. Note that $l_{0} \neq 0$. In fact a single integer $l$ in the range ]0,n-1] satisfies the previous condition: assume that $l$ and $l^{\prime}$ are solutions, that is $l p=s q-\mu$ and $l^{\prime} p=s^{\prime} q-\mu$. Then $l^{\prime}=l-\left(s^{\prime}-\right.$ $s) q / p$. Since $p$ and $q$ are coprimes, $s^{\prime}-s$ is a multiple of $p$ and then $l^{\prime}-l$ is a multiple of $q$, which proves the previous assertion. Therefore for $l_{0} p=s q-\mu$,

$$
F^{*}\left(\theta_{\lambda+\mu}\right) F\left(\theta_{\lambda}\right)=\frac{N^{2}}{q} e^{i \pi\left(l_{0}\left(\left(l_{0}+1\right) p-2 \lambda\right)\right) / q} .
$$

$$
<F^{*}\left(\theta_{\lambda+\mu}\right) F\left(\theta_{\lambda}\right)>_{\lambda}=\frac{N^{2}}{q} e^{i \pi \frac{l_{0}\left(l_{0}+1\right) p}{q}} \sum_{\lambda \bmod q} e^{-i 2 \pi \frac{l_{0} \lambda}{q}}
$$

This expression vanishes since $l_{0}$ is not a multiple of $q$ $\left(l_{0} \neq 0\right.$ and $\left.0 \leq l_{0} \leq q-1\right)$. Finally

$$
\left\langle F^{*}\left(2 \pi \frac{\lambda+\mu}{q}\right) F\left(2 \pi \frac{\lambda}{q}\right)\right\rangle_{\lambda}=\frac{N^{2}}{q} \delta(\mu \bmod q)
$$

where $\delta(\mu \bmod q)=1$ when $\mu$ divides $q$ and 0 else.

This peculiar correlation property could also have been demonstrated from the direct expressions of $F\left(\theta_{\lambda}\right)$ (Eq. (19) to (21)). The lack of average correlation between different pulses of the pulse train is in fact a general feature of Gauss sequences [45].
[1] H.F. Talbot, Philos. Mag. 9, 401 (1836).

[2] P. Latimer, R. F. Crouse, Appl. Opt. 31, 80 (1992);

[3] M.V. Berry, S. Klein, J. Mod. Opt. 43, 2139 (1996).

[4] S. Matsutani, Y. Onishi, Found. of Phys. 16, 325 (2003).

[5] I.S. Averbukh, N.F. Perelman, Phys. Lett. A 139, 449 (1989).

[6] M.J.J. Vrakking, D.M. Villeneuve, and A. Stolow, Phys. Rev. A 54, 37 (1996).

[7] R.W. Robinett, Phys. Rep. 392, 1 (2004).

[8] G. Della Valle et al. Phys. Rev. Lett. 102, 180402 (2009).

[9] S. Lang, Algebraic Number Theory (New York: AddisonWesley, 1970).

[10] D. Mumford, Tata Lectures on Theta I (Birkhäuser 1983).

[11] J. Marklof, Duke. Math. J. 97127 (1999).

[12] W. Merkel et al., Fortschr. Phys. 54, 856 (2006).

[13] M. Mehring, K. Mueller, I. S. Averbukh, W. Merkel, and W. P. Schleich, Phys. Rev. Lett. 98, 120502 (2007).

[14] D. Bigourd, B. Chatel, W. P. Schleich, and B. Girard, Phys. Rev. Lett. 100, 030202 (2008).

[15] A. A. Rangelov, J. Phys. B: At. Mol. Opt. Phys. 42 021002 (2009).

[16] V. Tamma et al., Phys. Rev. A 83, 020304 (2011).

[17] S. Wolk et al., New J. Phys. 13, 1 (2011).

[18] G. C. Valley, Opt. Exp. 15, 1955 (2007).

[19] G. V. Hartland, Annu. Rev. Phys. Chem. 57, 403 (2006).

[20] M. Pelton et al., Nature Nanotech. 4, 492 (2009).

[21] J. Azana, M. A. Muriel, Opt. Lett. 24, 1672 (1999).

[22] J. Azana, M. A. Muriel, IEEE. J. Sel. Top. in Quant. Elec., 7, 728 (2001).

[23] J. Azana et al., IEEE Photon. tech. Lett. 15, 413 (2003).
[24] F. V. Kowalski et al., Appl. Phys. Lett. 50, 711 (1987).

[25] P. D. Hale, F. V. Kowalski, IEEE. J. Quant. Elec. 26, 1845 (1990).

[26] P. Coppin, T. G. Hodgkinson, Elec. Lett. 26, 28 (1990).

[27] G. L. Bourdet, Appl. Opt. 42, 5457 (2003).

[28] H. Y. Ryu et al. Opt. Exp. 15, 11396 (2007).

[29] M. P. Nikodem et al. Opt. Exp. 17, 3299 (2009).

[30] M. W. Phillips, G. Y. Liang, J. R. M. Barr, Opt. Comm. 100, 473 (1993).

[31] L. P. Yatsenko et al., Opt. Comm. 236, 183 (2004).

[32] H. Guillet de Chatellus et al., Opt. Exp. (2013), in press.

[33] M. V. Berry, J. Goldberg, Nonlinearity 1, 1 (1988).

[34] M. A. Foster et al., Nature 456, 81 (2008).

[35] H. C. Rosu et al., Int. J. Mod. Phys. B 20, 1860 (2006).

[36] J. H. Hannay, M. V. Berry, Physica 1D, 267 (1980).

[37] H. Guillet de Chatellus et al., Opt. Comm. 284, 4965 (2011).

[38] F. V. Kowalski et al., Opt. Lett. 13, 622 (1988).

[39] H. Guillet de Chatellus, J.-P. Pique, Opt. Comm. 283, 1971 (2010).

[40] D. J. Newman, Proc. Am. Math. Soc. 16, 1287 (1965).

[41] R. Loudon, "Quantum Theory of Light", 3rd ed. (Oxford U. Press, New York, 2000), Chap. 3.

[42] Y. Shih, IEEE J. Sel. Top. in Quant. Elec. 13, 1016 (2007).

[43] J. Pumplin, J. Acoust. Soc. Am. 78, 100 (1985).

[44] M. R. Schroeder, IEEE Trans. Inf. Theo. 85 (1970).

[45] M. R. Schroeder, "Number Theory in Science and Communications", 5th ed. (Springer, Berlin Heidelberg 2009), Chap. 16.

[46] A. Khilo et al., Opt. Exp. 20, 4454 (2012). 
[47] M. F. Brandl, O. D. Muecke, Opt. Lett. 32, 4223 (2010). 